\title{
Anti-inflammatory activity of Indonesian nutmeg seeds (Myristica fragrans Houtt): A topical gel formulation
}

\author{
Azis Ikhsanudin, Lolita Lolita, Daniar Dyanas Rais
}

Faculty of Pharmacy, Universitas Ahmad Dahlan, Yogyakarta, Indonesia

\begin{tabular}{l}
\hline \hline Article Info \\
\hline Article history: \\
Received Mar 1, 2021 \\
Revised Jun 6, 2021 \\
Accepted Jun 23, 2021 \\
\hline
\end{tabular}

\section{Keywords:}

Anti-inflammatory

Herbal gel

Myristicin

Nutmeg seed

\begin{abstract}
Herbal medicines have been shown as anti-inflammatory with potentially lesser side effects. The active compound of nutmeg seed is proven to accelerate the healing process of inflammation. This study aimed to evaluate the gel formulation of Indonesian nutmeg (Myristica fragrans Houtt) seed extract for anti-inflammatory activity. A true experimental post test only with control group design was used in this study. The gel was formulated with various concentrations of nutmeg seed extract, namely formulations F1 (0\%), F2 (2\%), F3 (4\%), F4 (8\%), and F5 (12\%). Analysis of variance (ANOVA) followed by the least significant difference (LSD) methods were performed with SPSS version 22. The results showed that all formulas had an opaque physical appearance, brownish-yellow color, soft texture, and aromatic odor. The increase of extract concentration in gel formula will affect the adhesion and spreadability. F5 showed the highest anti-inflammatory activity compared to other groups. This formula was generally identified as having a good physical appearance, homogeneity, and stability with a $\mathrm{pH}$ value of $6.16 \pm 0.24$, adhesiveness of $51.12 \pm 0.15 \mathrm{sec}$, and a spreadability of $19.54 \pm 0.12 \mathrm{~cm} 2$. Therefore, Indonesian nutmeg has the potential to be wellacceptable as a candidate for topical anti-inflammatory agents in global health benefits.
\end{abstract}

This is an open access article under the CC BY-SA license.

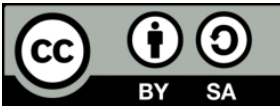

\section{Corresponding Author:}

Azis Ikhsanudin

Faculty of Pharmacy

Universitas Ahmad Dahlan

Prof Dr Soepomo Warungboto Umbulharjo, Yogyakarta, Indonesia

Email: azis.ikhsanudin@pharm.uad.ac.id

\section{INTRODUCTION}

Inflammation is a tissue-response cascade defense mechanism for specific inflammatory stimuli or pathogenic infections. This inflammatory reaction involves different cellular and vascular pathways with specific humoral secretions to prevent damage and restore tissue function [1]. Specific receptor cells could also trigger various pro and anti-inflammatory cytokines such as IL-1b, IL-6, IL-1a, and TNF- $\alpha$, which modulate neutrophil migration to endothelial cells. Neutrophils will be released into circulation upon encountering multiple danger signals by producing inflammatory cytokines. Moreover, monocytes will differentiate into tissue macrophages by phagocytosis of damaged cells and other invading pathogens [2], [3].

The drugs most commonly used for alleviating acute and chronic inflammation are non-steroidal anti-inflammatory drugs (NSAIDs) and steroids (corticosteroids) [4], [5]. These medications are typically used in combination because of their slightly distinct actions in granulation tissue. NSAIDs inhibit the cyclooxygenase enzymes (COX-1 and COX-2) responsible for regulating many cellular processes during the inflammatory response [6]. Steroids could suppress inflammation intensely by binding to receptors that 
control phospholipase A2, COX-2, iNOS, and interleukin. Unfortunately, two classes of drugs should be taken with caution because of the potential adverse events, e.g., gastrointestinal bleeding, myocardial infarction, and kidney disorders [4], [7].

The development of a plant-based drug is currently the primary alternative approach regarding the long-term risk of using anti-inflammatory drugs. Under these circumstances, medicinal plants have significantly rediscovered cellular pathways to provide potent active compounds as therapeutic phytochemicals [8]. Furthermore, the market for herbal medicines accounts for $83 \%$ worldwide in the treatment of inflammatory diseases. It is estimated that it will reach a value of approximately more than $95 \%$ in the forthcoming years due to increased revenues from these preparations. Indonesia is the largest producer and exporter of nutmeg in the global market. Overall export volume tended to increase by an average of 3.07\% per year during 2009-2018 period [9]. Indonesia nutmeg (Myristica fragrans Houtt) is of good quality due to its low and almost non-existent aflatoxin content [10]. The myristin content in Indonesian nutmeg was $8.72 \%$ higher than Indian nutmeg, which was only 3.8\% [11], [12].

A feasibility study reports that myristicin has been recognized in pharmacological mechanisms as a potent anti-inflammatory activity. Several studies have shown that nutmeg seed essential oil containing myristicin and aluminometasilicate was useful for anti-inflammatory activity. It works by inhibiting the biosynthesis of prostaglandin in the human colon. The high myristicin content in nutmeg could play a role in reducing TNF- $\alpha$ level. Myristicin also demonstrates a similar mechanism as non steroid anti-inflammatory drug's (NSAIDs) such as indomethacin and aspirin [13], [14]

Topical drug delivery systems are gaining popularity in developing local and systemic drug delivery systems. This system could avoid gastrointestinal irritation, overcome the "first pass" effect and maximize drug concentrations at receptor site [15]. Furthermore, the gel has biodegradable, biocompatible, consistent properties, reasonable penetration rates, and longer retention time [16]-[18]. Previous studies have reported the topical gel formulation of ethanol extract of nutmeg using the albumin denaturation method. This finding showed that nutmeg extract gel could inhibit albumin's denaturation higher than the marketed synthetic drug gels [19]. In our recent study, we used the carrageenan-induced rat paw edema model to evaluate antiinflammatory activity. The measurement of anti-inflammatory activity was based on the increase in the volume of rat leg edema, and the percentage was calculated based on the area under curve (AUC) value. This approach is more representative for determining the actual anti-inflammatory activity for topical drug preparations. Therefore, our study aimed to design and evaluate Indonesian nutmeg seed extract's topical anti-inflammatory activity using the carrageenan-induced rat paw edema model. This study's significance is expected to produce potent plant-based anti-inflammatory properties based on good efficacy and acceptance standards.

\section{RESEARCH METHOD}

The research used true experimental post test only with control grup design. This study aimed to cmpare the anti-inflammatory activity between formulas groups of topical herbal gel from Indonesian nutmeg (Myristica fragrans Houtt) seed extract.

\subsection{Plant materials and chemical reagents}

We collected fresh nutmeg seeds from Malikrubu Regency, Ternate, North Maluku, Indonesia. The plant was identified and approved by the Biology Laboratory of the Faculty of Mathematics and Natural Sciences, Ahmad Dahlan University. The chemical reagents such as distilled water, carbopol 940, methylparaben, propylene glycol, and triethanolamine were obtained through Brataco, Ltd, Indonesia. Voltaren gel containing $1 \%$ diclofenac sodium was obtained from the community pharmacist store, Ahmad Dahlan University. All of the reagents were of analytical and pharmaceutical grade.

\subsection{The extraction of indonesian nutmeg seed}

The purpose of the extraction was to preserve myristicin as an anti-inflammatory bioactive compound in nutmeg seed. Sample preparation was accomplished by peeling the fresh nutmeg using sharp stainlesssteel knives. Afterward, nutmeg seeds should be washed and dried in an oven at $40^{\circ} \mathrm{C}$ for 48 hours. The dried nutmeg seed should be ground to $0.4 \mathrm{~mm}$ in size using an electric blender to enhance the contact surface. The seed extract was prepared by maceration using $70 \%$ ethanol as a solvent for three days at room temperature. About 500g of nutmeg seed powdered was loaded using one liter of $70 \%$ ethanol for maceration, followed by stirring with a magnetic stirrer for two hours. The supernatant liquid mixture was poured onto filter paper and allowed to stand for 24 hours before being filtered. The whole liquid was filtered using a Buchner porcelain funnel. This treatment procedure was repeated for $3 \times 24$ hours to obtain a clear filtrate. Further, a gradual evaporation process was performed to obtain a concentrated extract [20]. 


\subsection{Topical gel formulation of indonesian nutmeg seed extract}

The gels were formulated using different concentration of nutmeg seeds extract ethanol $(0 \% ; 2 \%$; $4 \%$; $8 \%$ and 12\%), carbopol $940(1.5 \%)$, ethanol 96\% (4\%), propylene glycol (10\%), glycerin $(20 \%)$, triethanolamine $(0.8 \%)$, methylparaben $(0.1 \%)$, polysorbate $80(0.8 \%)$, and purified water (q.s to $100 \%)$. We prepared the gel-based mixture by mixing carbopol in purified water and maintained it with magnetic stirring until homogeneous (mixture A). Propylene glycol and triethanolamine are dissolved in mixture A to obtain a swollen gel with a pH of 5-6 (mixture B). Afterward, add the nutmeg ethanol extract to mixture B and stir with methylparaben to form a stable and homogeneous gel. Furthermore, the nutmeg extract gels must be stored in a tightly-closed container for 24 hours until the bubbles are removed [19]. The ethanol extract gel formula of nutmeg seed is shown in Table 1.

Table 1. Gel formula of ethanol extract nutmeg seed

\begin{tabular}{cccccc} 
Ingredient & Formula (gram) \\
& F1 & F2 & F3 & 4 & F5 \\
\hline Nutmeg seed extract & 0 & 1 & 2 & 0.75 & 6 \\
Carbopol 940 & 0.75 & 0.75 & 0.75 & 2 & 0.75 \\
Ethanol 96\% & 2 & 2 & 2 & 5 & 2 \\
Propylenglycol & 5 & 5 & 5 & 10 & 5 \\
Glyserin & 10 & 10 & 10 & 0.4 & 10 \\
Trietanolamin & 0.4 & 0.4 & 0.4 & 0.05 & 0.4 \\
Methyl paraben & 0.05 & 0.05 & 0.05 & 0.4 & 0.05 \\
Polysorbate 80 & 0.4 & 0.4 & 0.4 & 50 & 0.4 \\
Purified water ad & 50 & 50 & 50 & 4 & 50 \\
Note: The positive control (F6) used voltaren 50 g gel containing 1\% diclofenac sodium
\end{tabular}

\subsection{Evaluation of formulated nutmeg seed gel}

The formulated nutmeg seed gel was characterized by specific physical properties such as organoleptic, $\mathrm{pH}$, spreadability, and adhesivity. This parameter has the function of assessing the quality of the gel in various formulas. Another parameter was to investigate the potential activity of the gel as an antiinflammatory. The test procedure was performed using the carrageenan-induced rat paw edema model. This acute inflammation model is well-accepted and has long been used to determine its potent anti-inflammatory effect.

\subsubsection{Organoleptic properties}

Gel formulations with or without nutmeg seed extract were evaluated organoleptically for color, texture, and homogeneity. These characteristics were performed by visual observation. The formulation consistency and the particle's coarseness were checked by texture and homogeneity parameters. To assess these parameters, we pressed a small amount of formulated gel between the thumb and the index finger.

\subsection{2. pH measurement}

Electrochemical $\mathrm{pH}$ measurement is used to determine the acidity or alkalinity through a digital $\mathrm{pH}$ meter (HANNA HI9813-6 Portable). The principle step was to weigh one gram of each gel formula and completely immersed it in the glass electrode. All measurements were taken three times, for which the average reading was recorded [21].

\subsubsection{Spreadability}

The prepared gel spreadability was intended to evaluate the gel's ability to spread and absorb on the skin. This technique was performed by measuring the diameter of spread of 1 gram of gel samples on two horizontal glass slides $(10 x 20 \mathrm{~cm} 2)$ after $60 \mathrm{sec}$. The standard upper plate for determining gel spreadability was $0.5 \mathrm{~g}$. Each gel formulation was recorded in triplicate [19].

\subsubsection{Adhesivity}

The purpose of gel adhesion testing was to determine the strength that separates the gel from the surface. A tensile adhesion test measures this by placing 2 grams of gel between two resin plates in the designated area. Then clamp the load of $1 \mathrm{~kg}$ on the glass plate for five minutes. The static resin plate was attached to the adhesion apparatus by applying a trigger load of 80 grams. Further, the evaluation was carried out to determine the length of time using a stopwatch until the resin plate runs out [22]. 


\subsubsection{In-vitro anti-inflammatory activity of nutmeg seed extract}

The study protocol has obtained ethical approval from Ahmad Dahlan University Ethics Committee with reference number 011502014. The anti-inflammatory properties of nutmeg seed gel products were measured using carrageenan-induce acute inflammation model in hind leg edema of male Wistar rat. The carrageenan-induced paw edema method is a well-defined model of acute inflammation involving various inflammatory mediators [23]. Initially, healthy Wistar strains (150-200 grams per each) were obtained from an animal breeding house, Faculty of Veterinary Medicine, Gadjah Mada University, Yogyakarta. Rats kept under laboratory conditions were fed twice daily with standard rat chow and water ad libitum. All rats were randomly allocated into six groups: one negative control group, four intervention groups, and one positive control group. Each group comprised at least five experimental animals. Before the experiment, the animal had to fast for 24 hours with access to water. $50 \mu \mathrm{l}$ of $1 \% \lambda$-carrageenan solution (Sigma-Aldrich, Milan, Italy) was suspended in $1 \% \mathrm{NaCl}$ solution, which had been prepared one hour before the experiment. Furthermore, this solution should be injected into the plantar side of the rat's right hind paw. About 0.2 grams of nutmeg seed gel in a different formula was gently applied 50 times with the index finger after 15 minutes of carrageenan injection. The rat paw edema volume was measured immediately using a plethysmometer (model UGO BASILE S.R.L: 7141) at 15 minutes to three hours after carrageenan injection. The percentage of inflammation was calculated by the following formula:

$$
\% \text { Inflammatory }=\frac{\mathrm{V} 0-\mathrm{Vt}}{\mathrm{V} 0} \times 100 \%
$$

Note: Vo and Vt are the volumes of rat paw edema from the control group and experimental group.

\subsection{Data analysis}

The study results were reported descriptively on several test data such as organoleptic properties, $\mathrm{pH}$, adhesiveness, and spreadability. The statistical analysis was performed using One-Way ANOVA and followed by the LSD-post hoc test. A p-value less than 0.05 were considered statistically significant.

\section{RESULTS AND DISCUSSION}

We have successfully formulated herbal gel from the ethanol extract of nutmeg seed in various formulas. Our study demonstrated that the obtained nutmeg seed gel has a good physical appearance and an anti-inflammatory activity due to acute edema reduction. The detailed finding regarding physical characteristics and anti-inflammatory activity will be presented in the subsection below.

\subsection{Organoleptic properties}

As shown in Table 2, the nutmeg seed gel has a good cosmetic appearance with a soft texture, opaque, brownish-yellow in color, characteristic aromatic odor, and homogeneous without segregation. In general, the dense pigment of nutmeg seed contains more dyes to produce a brownish-yellow gel. Previous studies have also reported that the nutmeg seed gel's physical properties were brownish yellow, fragrant and homogeneous [22], [19]. The ethanolic extract does not dissolve completely in the gel bases due to the differences in solubility level. The appearance of nutmeg seed extract gel with various extract concentrations is shown in Figure 1.

Table 2. Organoleptic properties of nutmeg seed extract gels

\begin{tabular}{ccccccc}
\hline Formula & Physical appearance & Color & Odor & Texture & Homogenity & Segregation \\
\hline 1 & Transparant & Clear white & Typical of base & Soft & Homogenous & No \\
2 & Opaque & Brownih yellow & Typical of nutmeg oil & Soft & Homogenous & No \\
3 & Opaque & Brownish yellow & Typical of nutmeg oil & Soft & Homogenous & No \\
4 & Opaque & Brownish yellow & Typical of nutmeg oil & Soft & Homogenous & No \\
5 & Opaque & Brownish yellow & Typical of nutmeg oil & Soft & Homogenous & No \\
\hline
\end{tabular}




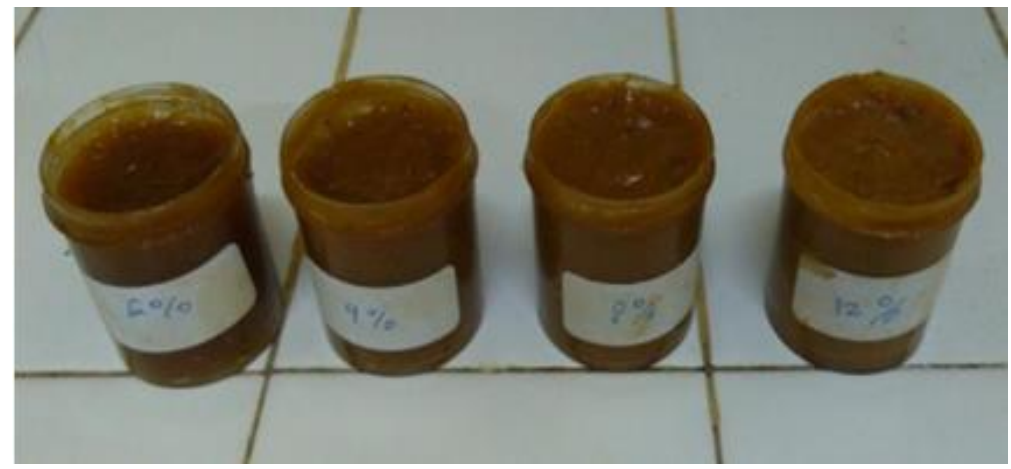

Figure 1. The physical appearance of nutmeg seed extract gel at F2 (2\%); F3 (4\%); F4 (8\%) and F5 (12\%) (From left to right)

\section{2. $\mathrm{pH}$ measurement}

Each nutmeg seed extract gel formula shows the following $\mathrm{pH}$ value F1(4.69 \pm 0.74$)$, F2 (5.38 \pm 0.78$)$, F3 (5.82 \pm 0.15$),$ F4 (6.33 \pm 0.13$),$ F5 $(6.16 \pm 0.24)$ and F6 $(5.87 \pm 0.12)$ as shown in Table 3. These pH values have met the standard criteria for topical skin administration ( $\mathrm{pH}$ ranged 4-7). $\mathrm{pH}$ value measurement could be related to the safety and efficacy of topical drug preparations. Gel acidity and alkalinity will affect skin irritation, penetration and stability of active compounds into the skin. The more acidic the $\mathrm{pH}$ value of gel will increase skin irritation, while the more alkaline it will cause dry skin [24], [22]. The statistic analysis using the LSD post hoc test showed a significant difference in $\mathrm{pH}$ value between F3 to F5 compared to F1 $(p<0.001)$. These results mean that the $\mathrm{pH}$ value will increase with the addition of nutmeg extract concentration in the gel base. Meanwhile, the $\mathrm{pH}$ value of F1 (gel base) was not significantly different from F2 (extract 2\%) ( $p=0.121)$. This defines that both formulas have a similar $\mathrm{pH}$ value. As a gelling agent, each end of carbopol's chain has an acidic carboxylic chemical structure when reacting to water. Carbopol 940 is easily ionized during the neutralization process with the addition of triethanolamine. Therefore, the interaction between carbopol 940 and triethanolamine could affect the $\mathrm{pH}$ stability based on the increase in extract concentration [25].

Table 3. The $\mathrm{pH}$ value of nutmeg seed extracts gel

\begin{tabular}{ccccccc}
\hline \multirow{2}{*}{ Formula } & \multirow{2}{*}{$\mathrm{pH}($ Mean \pm SD) } & F1 & p-value $(\mathrm{LSD}$ post hoc test) \\
& & $\mathrm{F} 2$ & $\mathrm{~F} 3$ & $\mathrm{~F} 4$ & $\mathrm{~F} 5$ \\
\hline $\mathrm{F} 1$ & $4.69 \pm 0.74$ & - & 0.121 & $0.020^{*}$ & $0.002^{*}$ & $0.050^{*}$ \\
$\mathrm{~F} 2$ & $5.38 \pm 0.78$ & 0.121 & - & 0.306 & $0.041^{*}$ & 0.084 \\
$\mathrm{~F} 3$ & $5.82 \pm 0.15$ & $0.020^{*}$ & 0.306 & - & 0.234 & 0.419 \\
$\mathrm{~F} 4$ & $6.33 \pm 0.13$ & $0.002^{*}$ & $0.041^{*}$ & 0.236 & - & 0.680 \\
$\mathrm{~F} 5$ & $6.16 \pm 0.24$ & $0.005^{*}$ & 0.084 & 0.419 & 0.680 & - \\
\hline Note: * p-value less than 0.05 & & & & &
\end{tabular}

\subsection{Adhesivity}

Penetration of the active compound gel into the skin will increase along with the enhancement of gel adhesivity properties. The results showed that the average adhesion score of nutmeg seed extract gel was F1

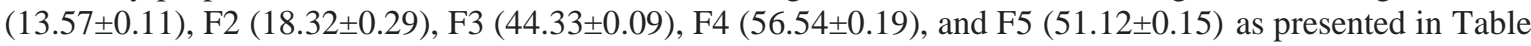
4. Based on a one-way ANOVA test followed by LSD statistical analysis, the nutmeg extract gel of F4 was significantly greater in adhesion than other formulas $(\mathrm{p}<0.005)$. Furthermore, the formulation of F5 with $12 \%$ nutmeg seed ethanol extract showed a decrease in adhesiveness compared to F4. This decrease might be due to the $\mathrm{pH}$ value being smaller at F5 than F4. The cross-linking between carbopol and other molecules, such as solvents, could also result in ionic attraction, and increasing the gel's viscosity. In acid conditions, increasing the gel's viscosity will have an impact on decreasing the adhesive properties.

Table 4. Adhesive properties of the nutmeg seed extract gel

\begin{tabular}{ccccccc}
\hline \multirow{2}{*}{ Parameter } & \multicolumn{4}{c}{ Mean \pm SD (second) } & \multicolumn{2}{c}{ p-value } \\
& F1 & F2 & F3 & F4 & F5 & \\
\hline Adhesivity test (sec) & $13.57 \pm 0.11$ & $18.32 \pm 0.29$ & $44.33 \pm 0.09$ & $56.54 \pm 0.19$ & $51.12 \pm 0.15$ & 0.000 \\
\hline
\end{tabular}




\subsection{Spreadability}

As shown in Table 5, the average spreadability of the nutmeg gel formulations showed F1 (9.38 \pm 0.65$), F 2$ (11.54 \pm 0.21$),$ F3 (13.39 \pm 0.06$)$, F4 (14.85 \pm 0.34$)$, and F5 (19.54 \pm 0.12$)$. These findings concluded that increasing the gel extract concentration would affect the broadest spreadability of the gel. The formula F5 (12\%) has the greatest spreadability compared to other formulas. The statistical analysis results through one-way ANOVA stated a significant difference in spreadability between all formulas $(\mathrm{p}<0.005)$. Carbopol 940, an acrylic polymer gelling agent, could regulate the viscosity in $1-2 \%$ concentrations by producing a three-dimensional matrix to form a viscous gel. The spreadability of the gel formula was highly dependent on the viscosity of the carbopol. The less viscous gelling agent will enhance the spreadability of the gel. The swelling gel process occurs due to the solvent penetration, leading to the cross-linked polymer network to maintain the dosage form and the binding drug particles. However, the alkaline environment $\mathrm{pH}$ will increase the density of negative charge, resulting in the gel swollen and facilitating the drug's release [16], [25]. Furthermore, the addition of nutmeg seed extract to the gel base will also affect the formation of hydrogen bonds in the physical cross-linking, which influences a reduced viscosity [16].

Table 5. Spreadability properties of the nutmeg seed extract gel

\begin{tabular}{ccccccc}
\hline \multirow{2}{*}{ Parameter } & \multicolumn{3}{c}{ Mean \pm SD $\left(\mathrm{cm}^{2}\right)$} & \multicolumn{2}{c}{ p-value } \\
& F1 & F2 & F3 & F4 & F5 & \\
\hline Spreadability test $(\mathrm{cm})$ & $9.38 \pm 0.65$ & $11.54 \pm 0.21$ & $13.39 \pm 0.06$ & $14.85 \pm 0.34$ & $19.54 \pm 0.12$ & 0.000 \\
\hline
\end{tabular}

\subsection{In-vitro anti-inflammatory activity of nutmeg seed extract gel}

The evaluation of the nutmeg seed herbal gel's anti-inflammatory activity was observed within 180 minutes as shown in Figure 2. During this time, carrageenan will significantly increase TNF- $\alpha$, IL-1 $\beta$, PGE2, iNOS, and COX-2 proteins in peripheral leg inflammation. Carrageenan-induced leg edema follows a model of acute inflammation which consists of two phases: first, which was detected after about 1 hour and was called the fast phase, with the release of histamine and serotonin, and the second stage was called the late phase with the mediators (kinins, prostaglandins) released after two and three hours, respectively [26]. A previous study suggested that intraplantar carrageenan injection will stimulate rat paw edema and release inflammatory mediators within $180 \mathrm{~min}$ [27]. This study is in line with our findings that decreased edema volume also occurred at 180 minutes after intraplantar carrageenan administration.

Figure 2 shows that the formulation of nutmeg seed extract gel has anti-inflammatory effects. It can be proven that the percentage volume of edema in each formula (F2 to F6) was lower than F1 (gel-based negative control). The anti-inflammatory activity of F5 (12\%) was higher than that of F2 (2\%) and F3 (4\%). Furthermore, F4 (8\%) and F5 (12\%) had anti-inflammatory activity similar to F6 (positive control). This antiinflammatory activity is influenced by the main compound contained in the nutmeg, namely myristicin. The active compound of nutmeg seed extract, myristicin, has a vital function as an anti-inflammatory by inhibiting chemokines, cytokines, nitrous oxide, and double-stranded growth factors RNA (dsRNA), which are stimulated by macrophages through calcium [28]. Other quercetin compounds also have antiinflammatory activity by inhibiting the secretion of TNF- $\alpha$, IL-6, IL-1 $\beta$, and nitric oxide (NO) [29].

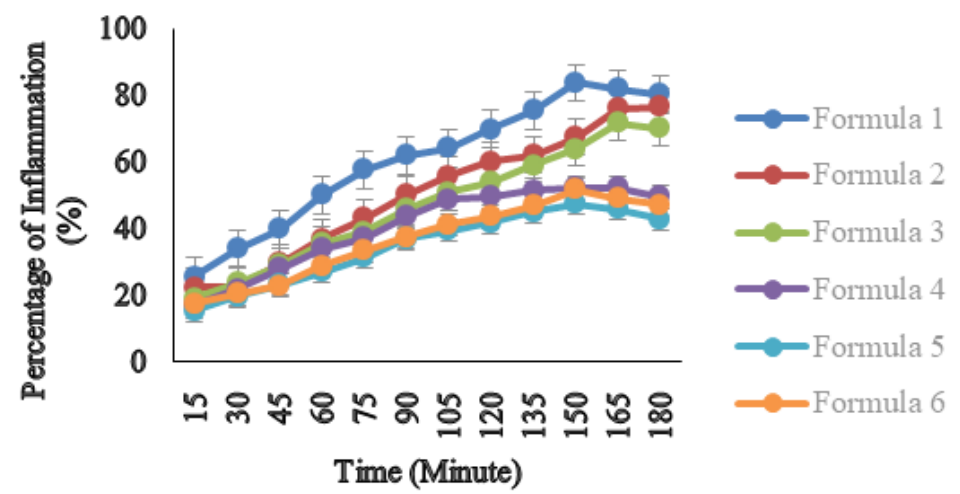

Figure 2. Inflammatory activity of nutmeg seed extracts gels 
The volume increase in rat leg edema was used to calculate each formula's anti-inflammatory percentage based on the AUC value. The calculated AUC value and the percentage of anti-inflammatory can be seen in Table 6. Based on the LSD post hoc test analysis reported that the anti-inflammatory activity of F4, F5 did not have a significant difference compared to F6 (positive control). It was concluded that F4 and F5 had similar anti-inflammatory activity as the positive control (1\% diclofenac sodium gel in the marketed product). Furthermore, the anti-inflammatory activity of F5 (44.92\%) was higher significant difference compared to F4 $(37.87 \%)$ with $p=0.025$ The AUC calculation results showed that F5 had the lowest AUC value (5803.72) with the highest percentage of anti-inflammatory activity $(44.92 \%)$ compared to other formulas (F1 to F5).

Table 6. The percentage of anti-inflammatory activity of nutmeg extract gel was compared between groups

\begin{tabular}{ccccccccc}
\hline \multirow{2}{*}{ Formula } & \multirow{2}{*}{ AUC } & \multirow{2}{*}{ Anti-inflammatory activity $(\%)$} & \multicolumn{4}{c}{ p-value (LSD post hoc test) } \\
& & & F1 & F2 & F3 & F4 & F5 & F6 \\
\hline F1 & 10536.94 & 0 & - & $0.000^{*}$ & $0.000^{*}$ & $0.000^{*}$ & $0.000^{*}$ & $0.000^{*}$ \\
F2 & 9304.97 & 11.69 & $0.000^{*}$ & - & $0.000^{*}$ & $0.000^{*}$ & $0.000^{*}$ & $0.000^{*}$ \\
F3 & 7919.26 & 24.84 & $0.000^{*}$ & $0.000^{*}$ & - & $0.000^{*}$ & $0.000^{*}$ & $0.000^{*}$ \\
F4 & 6546.62 & 37.87 & $0.000^{*}$ & $0.000^{*}$ & $0.000^{*}$ & - & $0.025^{*}$ & 0.423 \\
F5 & 5803.727 & 44.92 & $0.000^{*}$ & $0.000^{*}$ & $0.000^{*}$ & $0.025^{*}$ & - & 0.124 \\
F6 & 6295.140 & 40.25 & $0.000^{*}$ & $0.000^{*}$ & $0.000^{*}$ & 0.423 & 0.124 & - \\
\hline
\end{tabular}

The highest anti-inflammatory activity of F5 was due to the gel's spreadability and its adhesion to the skin surface. F5 has a good adhesiveness of $(51.12 \pm 0.15) \mathrm{sec}$ with a spreadability of $(19.54 \pm 0.12) \mathrm{cm}$. The topical drug penetration into the skin was affected by the length of time the gel is in contact with the skin surface. F5 has the highest concentration of nutmeg seed extract (12\%) than other formulas (F2 to F4). The high content of myristicin in the F5 gel formula will improve its anti-inflammatory effect by enhancing the drug permeation into the skin. Besides, F5 also had the optimal ternary system composition between ethanol, propylene glycol, and purified water. Propylene glycol (PG) as an enhancer will affect the drug absorption into the skin [30]. A study of topical ibuprofen on human skin reported that ethanol's ternary solvent system, propylene glycol, and water had higher permeability into the skin than the binary solvent system. The ternary system will increase the solubility partition and membrane permeability partitioning by maximizing the active drug flux [31].

\section{CONCLUSION}

These present study indicates that nutmeg gel extract (Myristica fragrans Houtt) with 12\% ethanolic extract concentration has a good physical appearance and potent anti-inflammatory activity than other formulas and marketed product. Further study should implement a novel pharmaceutical technology in topical drug delivery system regarding to enhance the anti-inflammation activity. Hence, Indonesian nutmeg seed extract could be a potential candidate as anti-inflammatory in topical gel dosage form application.

\section{REFERENCES}

[1] S. Kany, J. T. Vollrath, and B. Relja, "Cytokines in inflammatory disease," Int. J. Mol. Sci., vol. 20, no. 23, pp. 131, 2019, doi: 10.3390/ijms20236008.

[2] Y. Su, J. Gao, P. Kaur, and Z. Wang, "Neutrophils and macrophages as targets for development of nanotherapeutics in inflammatory diseases," Pharmaceutics, vol. 12, no. 12, pp. 1-24, 2020, doi: 10.3390/pharmaceutics12121222.

[3] J. Prado et al., "Cytokine receptor clustering in sensory neurons with an engineered cytokine fusion protein triggers unique pain resolution pathways," Proc. Natl. Acad. Sci. U. S. A., vol. 118, no. 11, pp. 1-12, 2021, doi: 10.1073/pnas.2009647118.

[4] M. Del Grossi Moura, L. C. Lopes, M. T. Silva, S. Barberato-Filho, R. H. L. Motta, and C. De Cássia Bergamaschi, "Use of steroid and nonsteroidal anti-inflammatories in the treatment of rheumatoid arthritis: Systematic review protocol," Med. (United States), vol. 97, no. 41, 2018, doi: 10.1097/MD.0000000000012658.

[5] A. Lahoti, B. S. Kalra, and U. Tekur, "Evaluation of the analgesic and anti-inflammatory activity of fixed dose combination: non-steroidal anti-inflammatory drugs in experimental animals.," Indian J. Dent. Res., vol. 25, no. 5, pp. 551-554, 2014, doi: 10.4103/0970-9290.147071.

[6] C. Gunaydin and S. S. Bilge, "Effects of nonsteroidal anti-inflammatory drugs at the molecular level," Eurasian J. Med., vol. 50, no. 2, pp. 116-121, 2018, doi: 10.5152/eurasianjmed.2018.0010.

[7] P. Kuropakornpong, A. Itharat, S. Panthong, S. Sireeratawong, and B. Ooraikul, "In Vitro and in Vivo AntiInflammatory Activities of Benjakul: A Potential Medicinal Product from Thai Traditional Medicine," Evidence- 
based Complement. Altern. Med., vol. 2020, 2020, doi: 10.1155/2020/9760948.

[8] A. G. Atanasov et al., "Natural products in drug discovery: advances and opportunities," Nat. Rev. Drug Discov., vol. 20, no. 3, pp. 200-216, 2021, doi: 10.1038/s41573-020-00114-z.

[9] H. Juliani Purba, E. Supriadi Yusufi, and J. Hestina, "Performane and Competitiveness of Indonesian Nutmeg in Export Market," E3S Web Conf., vol. 232, p. 02018, 2021, doi: 10.1051/e3sconf/202123202018.

[10] J. Marmis, N. S. Tongkeles, Lady Lengkey, and S. Ratulangi, "the Quality of Nutmeg Seeds and Mace," J. LPPM Bid. SAINS DAN Teknol., vol. 2, no. 1, pp. 111-121, 2015.

[11] K. Karmanah, S. Susanto, W. D. Widodo, and E. Santosa, "The Fruit Characteristics of Ambon Forest Nutmeg (Myristica fatua Houtt) and Banda Nutmeg (Myristica fragrans Houtt)," J. Ilmu Pertan. Indones., vol. 25, no. 2, pp. 292-300, 2020, doi: 10.18343/jipi.25.2.292.

[12] I. P. S. Kapoor, B. Singh, G. Singh, C. S. De Heluani, M. P. De Lampasona, and C. A. N. Catalan, "Chemical composition and antioxidant activity of essential oil and oleoresins of nutmeg (Myristica fragrans Houtt.) fruits," Int. J. Food Prop., vol. 16, no. 5, pp. 1059-1070, 2013, doi: 10.1080/10942912.2011.576357.

[13] I. Matulyte et al., "The essential oil and hydrolats from myristica fragrans seeds with magnesium aluminometasilicate as excipient: Antioxidant, antibacterial, and anti-inflammatory activity," Foods, vol. 9, no. 1, 2020, doi: 10.3390/foods9010037.

[14] W. K. Zhang et al., "Nutmeg oil alleviates chronic inflammatory pain through inhibition of COX-2 expression and substance P release in vivo," Food Nutr. Res., vol. 60, no. April, 2016, doi: 10.3402/fnr.v60.30849.

[15] M. M. Ghareeb, "Design and in vitro characterization of a topical nanoemulsionenriched hydrogel of econazole nitrate," J. Appl. Pharm. Sci., vol. 9, no. 1, pp. 51-57, 2019, doi: 10.7324/JAPS.2019.90108.

[16] S. A. A. Nabi, M. A. Sheraz, S. Ahmed, N. Mustaan, and I. Ahmad, "Pharmaceutical Gels : A Review," RADS J. Pharm. Pharm. Sci., vol. 4, no. 1, pp. 40-48, 2016.

[17] C. Tipa, M. T. Cidade, T. Vieira, J. C. Silva, P. I. P. Soares, and J. P. Borges, "Article a new long-term composite drug delivery system based on thermo-responsive hydrogel and nanoclay," Nanomaterials, vol. 11, no. 1, pp. 1-22, 2021, doi: 10.3390/nano11010025.

[18] R. Sánchez-Sánchez et al., "Development of Hydrogel with Anti-Inflammatory Properties Permissive for the Growth of Human Adipose Mesenchymal Stem Cells," J. Nanomater., vol. 2016, 2016, doi: $10.1155 / 2016 / 8654937$.

[19] S. Surendran and A. V. Vidyapeetham, "TOPICAL GEL AND ITS IN VITRO EVALUATION FOR ANTINFLAMMATORY," Int. J. Pharm. Technol., vol. 8, no. April 2016, pp. 1065-11076, 2018, [Online]. Available: https://www.researchgate.net/publication/301348769.

[20] E. F. Al-Jumaily and M. H. A. Al-Amiry, "Extraction and Purification of Terpenes from Nutmeg (myristica fragrans)," J. Al-Nahrain Univ. Sci., vol. 15, no. 3, pp. 151-160, 2012, doi: 10.22401/jnus.15.3.21.

[21] S. Karastogianni, S. Girousi, and S. Sotiropoulos, pH: Principles and Measurement, 1st ed., no. December. Elsevier Ltd., 2015.

[22] R. Aiyalu, A. Govindarjan, and A. Ramasamy, "Formulation and evaluation of topical herbal gel for the treatment of arthritis in animal model," Brazilian J. Pharm. Sci., vol. 52, no. 3, pp. 493-507, 2016, doi: 10.1590/s198482502016000300015.

[23] M. Kian, M. Azarbaijani and H. Soraya "Anti-inflammatory Effects of Memantine in Carrageenan-induced Paw Edema Model in Rats," Journal of Reports in Pharmaceutical Sciences, vol. 10, no. 1,pp. 60-65, 2021, doi: 10.4103/jrptps.JRPTPS

[24] S. M. Ali and G. Yosipovitch, "Skin pH: From basic science to basic skin care," Acta Derm. Venereol., vol. 93, no. 3, pp. 261-267, 2013, doi: 10.2340/00015555-1531.

[25] I. Giannopoulou, F. Saïs, and R. Thomopoulos, "Linked data annotation and fusion driven by data quality evaluation," Rev. des Nouv. Technol. l'Information, vol. E.28, pp. 257-262, 2015.

[26] C. W. Li et al., "Anti-inflammatory property of the ethanol extract of the root and rhizome of Pogostemon cablin (Blanco) benth," Sci. World J., vol. 2013, 2013, doi: 10.1155/2013/434151.

[27] J. C. Fehrenbacher, M. R. Vasko, and D. B. Duarte, "Models of inflammation: carrageenan-or complete freund's adjuvant (CFA)-induced edema and hypersensitivity in the rat," Curr. Protoc. Pharmacol., vol. 56, no. 1, pp. 4-5, 2012.

[28] J. Y. Lee and W. Park, "Anti-inflammatory effect of myristicin on RAW 264.7 macrophages stimulated with polyinosinic-polycytidylic acid," Molecules, vol. 16, no. 8, pp. 7132-7142, 2011, doi: 10.3390/molecules16087132.

[29] K. Dewi, B. Widyarto, P. Erawijantari, and W. Widowati, "In vitro study of Myristica fragrans seed (Nutmeg) ethanolic extract and quercetin compound as anti-inflammatory agent," Int. J. Res. Med. Sci., vol. 3, no. 9, pp. 2303-2310, 2015, doi: 10.18203/2320-6012.ijrms20150621.

[30] M. E. Lane, "Skin penetration enhancers," Int. J. Pharm., vol. 447, no. 1-2, pp. 12-21, 2013, doi: 10.1016/j.ijpharm.2013.02.040.

[31] R. M. Watkinson, C. Herkenne, R. H. Guy, J. Hadgraft, G. Oliveira, and M. E. Lane, "Influence of Ethanol on the Solubility, Ionization and Permeation Characteristics of Ibuprofen in Silicone and Human Skin," Skin Pharmacol. Physiol., vol. 22, no. 1, pp. 15-21, 2009, doi: 10.1159/000183922. 